\title{
Diagnosis of Subcutaneous Cysticercosis as a Cystic Mass Over Chest wall: A Case Report and Review of The Literature
}

\author{
Tanima Dwivedi ${ }^{1 *}$ and Reshma Davangeri ${ }^{2}$ \\ ${ }^{1}$ Department of Emergency Laboratory, Institute of Human Behaviour \& Allied Sciences, Delhi, India \\ ${ }^{2}$ Department of Pathology, KLE University's Jawaharlal Nehru Medical College, Belagavi, India
}

\begin{abstract}
Cysticercosis is the common tropical disease. It is usually present as a neurocysticercosis or intramuscular lesion, rarely may it present in subcutaneous tissue in the adult age group. Prevalence of asymptomatic subcutaneous nodules in India is $12.9-38 \%$. Subcutaneous nodule is usually occur as a part of the disseminated cysticercosis or as an isolated features. Here, we report a 45 year old male presenting with cyst over right chest wall but otherwise asymptomatic. A clinical diagnosis of epidermal cyst was made. Surgical excision was performed. Diagnosis of cutaneous cysticercosis was made by biopsy of subcutaneous nodule and patient was started on treatment.
\end{abstract}

Keywords: Subcutaneous Cysticercosis, cystic mass, chest wall

\section{Introduction}

Subcutaneous Cysticercosis is a tissue infection caused by Cysticercus cellulose, a larval form of the tape worm Taenia solium. . ${ }^{[1]}$ Humans acquire cysticercosis through fecal-oral contamination with $\mathrm{T}$. solium eggs from tape worm carriers. Cysticercosis can occur anywhere in the human body, but it is more common in the brain and skeletal muscles and uncommonly in subcutaneous tissues. It presents as asymptomatic subcutaneous nodules with prevalence of $12.9-38 \%$ in India. ${ }^{[1]}$ We here present a case of isolated subcutaneous cysticercosis without involvement of any other site.

\section{Case Report}

A 45 years male from Karnataka presented with an asymptomatic cyst over the right chest wall since 4 months. Patient was otherwise healthy without any systemic sign and symptoms. He was a farmer by occupation and lives in a lower middle income group. He consumed mixed diet.

Physical examination revealed one subcutaneous nodule over right chest wall just below the nipple in the mid clavicle wall. This nodule was well defined, non tender, firm, mobile with a smooth surface and measure $2 \times 2 \mathrm{cms}$. Other system examinations were unremarkable. A clinical differential diagnosis of epidermal cyst was given.

A complete blood count revealed hemoglobin- $13 \mathrm{~g} / \mathrm{dl}$, red blood cells - $6.5 \mathrm{million} / \mathrm{cc}$, white blood cells - 7500/cc, neutrophils - 65\%, lymphocytes - 30\%, eosinophils - 3\%, monocytes - $2 \%$, platelet count $-2 \mathrm{lakh} / \mathrm{cc}$, peripheral Smear: normocytic, normochromic blood picture . Serum biochemistry was also normal (fasting blood glucose-
$98 \mathrm{mg} / \mathrm{dl}$, blood urea $-28 \mathrm{mg} \%$, serum creatinine -0.8 $\mathrm{mg} / \mathrm{dl}$, serum sodium $-137 \mathrm{mmol} / \mathrm{L}$, serum potassium-4.2 $\mathrm{mmol} / \mathrm{L}$, serum calcium- $1.1 \mathrm{mmol} / \mathrm{L}$ ).Stool examination was negative for ova \& cyst and complete urine examination was within normal limits. Chest X-ray and ultrasonography abdomen were normal.

The lesion was surgically excised under general anesthesia and sent for histopathological examination. Specimen was received in Department of Pathology. On gross examination, it was a single, cystic, gray white lesion measuring $2 \times 1.8 \times$ $0.5 \mathrm{cms}$. Cut surface contain clear watery fluid and showed a gray white cystic cavity. (Figure 1)

On microscopic examination, the excised tissue showed a cystic cavity containing a larval form of Taenia solium showing cuticular layer, prominent sucker and translucent hooklets. Structure of cysticercosis cellulosae typical has duct-like invagination lined by homogeneous bilayered eosinophilic membrane. Cyst wall and outer fibrous tissue was thrown in multiple papillary projections. (Figure 2a, $2 \mathrm{~b}$ and $2 \mathrm{c}$ )

Patient was advised other investigation to rule out involvement of other organs by the disease. CT scan brain and Fundus examination was done which confirmed absence of disease in the respective sites. Patients was started on Albendazole $200 \mathrm{mg}$ TDS for 30 days. Six month follow up showed no recurrence.

\section{Discussion}

Campbell and Thomson in 1912 , reported the first case of cysticercosis involving the skin in India. ${ }^{1}$ Cysticercosis is a global problem mainly in the developing countries. It 


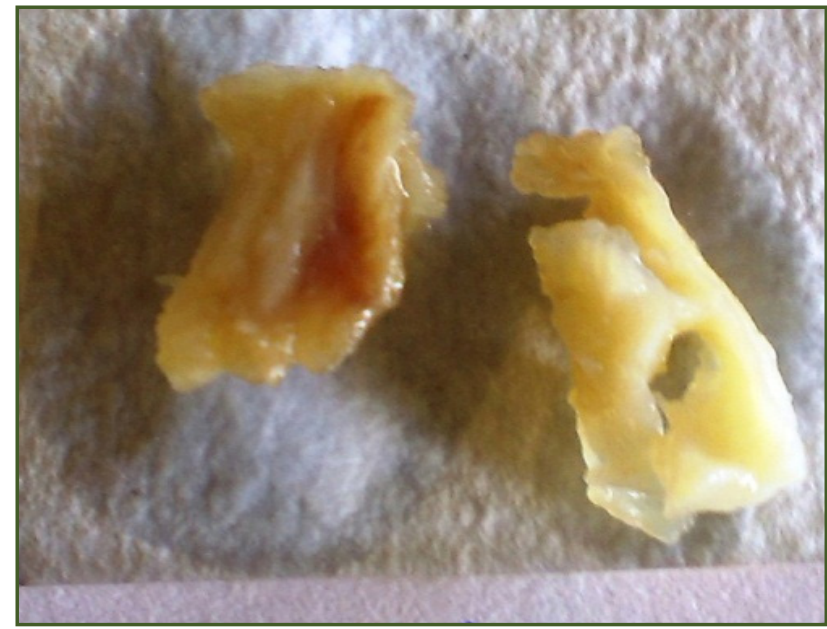

Fig. 1: Cut surface showed a cystic lesion containing clear watery fluid. It was a uniloculated, thin-walled cyst.

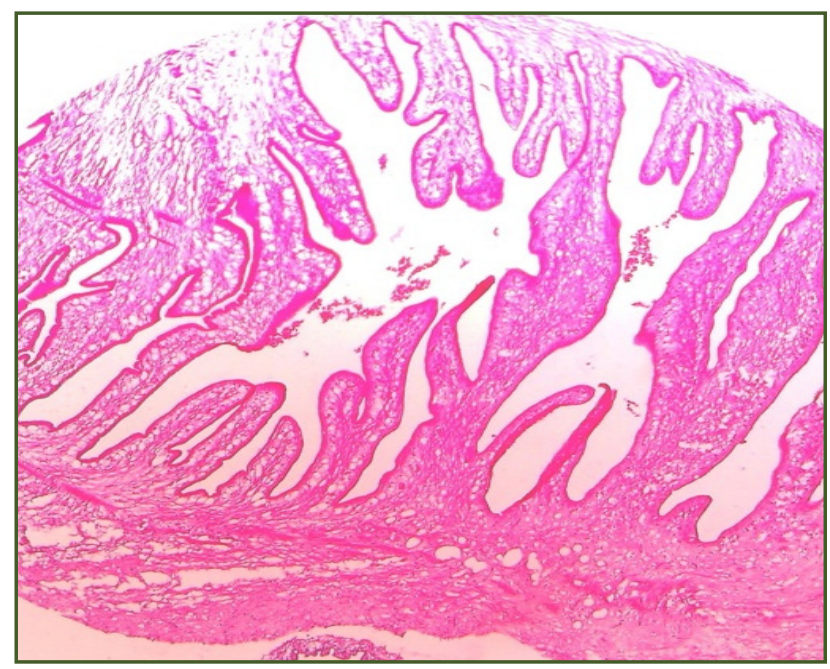

Fig. 2b: Duct-like invagination lined by bilayered eosinophilic membrane (H\&E at 10X).

has been eradicated from most of developed countries but it's still in Central and South America, South Asia, India and China. 50 million people in world are infected with the taeniasis/cysticercosis complex and 50,000 die from cysticercosis annually. Most infected individuals are aged between $20-50$ years. ${ }^{2}$

In India, because of disparities within the country in geography, ethnicity, religious rituals, income, standards of living, food habits, personal hygiene and level of education, which influence the disease burden and also lack of systematic population-based studies, disease is under reported. Consumption of pork is generally restricted to the lower socio-economic strata. Cysticercosis appears

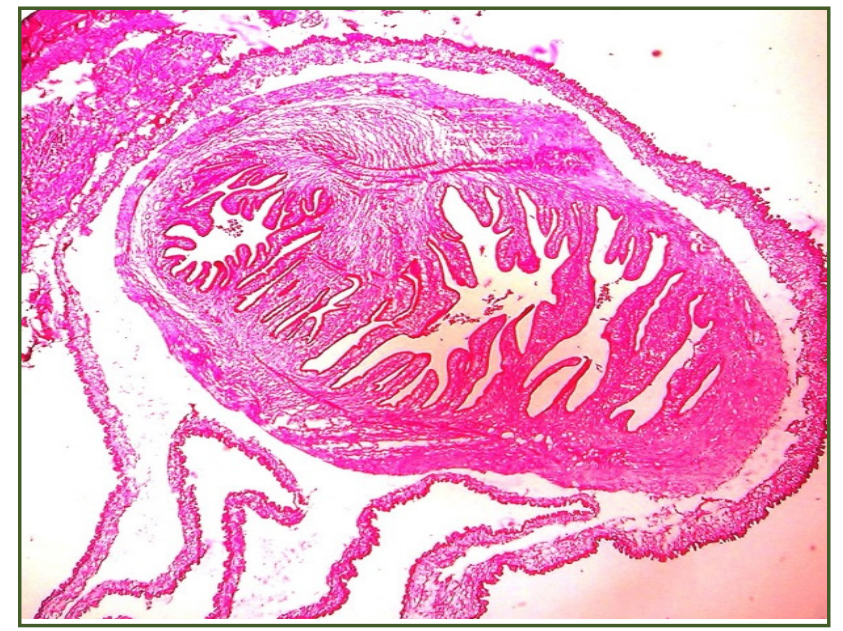

Fig. 2a: Structure of larval form of Taenia solium, Cysticercus cellulose, showing cuticular layer, prominent sucker and translucent hooklets. (H\&E at 10X ).

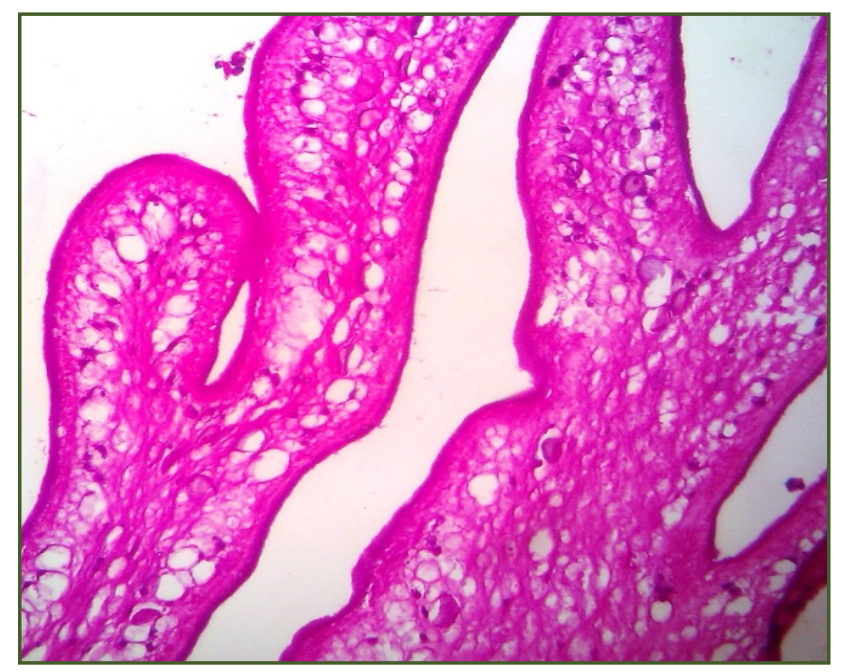

Fig. 2c: Photograph showing caudal end of larva lined by acellular, homogeneous eosinophilic membrane (H\&E, 40X)

to be more prevalent in the North India especially Bihar, Uttar Pradesh and Punjab because of high consumption of pork and low standard of living. State of Kerala has only few cases of cysticercosis probably due to the high level of education and standards of hygiene. In Jammu and Kashmir, as a Muslim majority State due to prohibition of pork consumption by religion results in low incidence of cysticercosis. ${ }^{3}$

Taenia solium has a complex two-host life cycle. Infection is acquired by ingestion of eggs through contaminated food, water or by autoinoculation. Incubation period varies from months to years. In cysticercosis, humans are only definitive hosts and pigs are the intermediate host. Both 
humans and pigs can act as intermediate hosts and harbour the larvae or cysticerci. Human's harbour the adult pork tapeworms in the small intestine and are the only source of cysticercosis for pigs as well as for the other humans. Infective eggs are detached from the adult tapeworm and are passed in the feces. As a result of improper fecal disposal, pigs consume the infected feces. Taenia solium eggs matures into oncospheres and metacestodes and lodge in the striated muscles and other tissues of pigs. These metacestodes evolve into larvaes (cysticerci). When human consume improper cooked infected pork, larvae evaginate and adhere to the intestinal mucosa. Cysticerci invade the intestine mucosa and enter the blood stream. Once they enter the blood stream of humans, they can lodge in any organ in the body such as the skeletal muscles, central nervous system, eye and subcutaneous tissue. ${ }^{4}$

Cysticercosis can occur anywhere in the human body, but it has predilection for the tissue like nervous tissue, eyes, skeletal muscles and subcutaneous tissues. Brain is the most common location accounting for $60-90 \%$ of all cases. ${ }^{1}$ Other site includes tongue, buccal mucosa etc. Involvement of the nervous tissue may cause severe morbidity but involvement of the subcutaneous tissue mainly causes cosmetic problems. Involvement of subcutaneous tissues often manifests as palpable subcutaneous nodules. Neurocysticercosis usually present as recurrent episodes of seizure, headache, focal neurological deficits, chronic meningitis, psychological disturbances, dementia, etc. Orbital cysticercosis may present as proptosis, diplopia and even loss of vision. Cysticercosis of the muscles may cause severe ache, pseudohypertrophy. ${ }^{2}$

A similar case of cysticercosis was reported by Siddewari et al. Patient complaint of seizures with two subcutaneous nodules one over left elbow and other over lateral aspect of left thumb. On Magnetic resonance imaging brain, it was suggestive of neurocysticerosis and biopsy of skin confirmed the diagnosis of subcutaneous cysticercosis. ${ }^{1}$ Rajan A et al did a study on soft tissue cysticercosis. They reported only 2 cases of subcutaneous cysticercosis out of 21 cases which occurred in soft tissue. They reported order of frequency of subcutaneous nodule is on upper arm, abdominal wall, chest wall, eyes, and neck. ${ }^{3}$
Diagnosis of soft tissue lesions requires identification of the Cysticercus cellulose, a larval form of the tape worm Taenia solium in the tissue by histopathological examination. Nigam JS et al reported a case of cysticercus cellulose on a 27-year-old female patient presented with a swelling on the right arm, by fine need aspiration cytology (FNAC).They reported that aspiration of clear fluid in palpable subcutaneous or intramuscular nodule is a strong clue for the diagnosis of cysticercosis. ${ }^{5}$

The treatment of cysticercosis depends on symptoms of the patients and accessibility of lesion. For symptomatic patients drugs like Praziquantel and/or Albendazole are effective. Treatment of choice in solitary accessible lesion is surgical excision. ${ }^{3}$

\section{Conclusion}

Cysticercosis of brain and skeletal muscle are relatively common than it's subcutaneous location. Therefore, it should be considered as differential diagnosis of subcutaneous swelling in the adult age group. Detail medical examination of all proven cases should be carried out, to rule out presence of parasite in any other site. Cysticercosis is considered as a "biological marker" for the social and economic development. Since, cysticercosis is a preventable and eradicable disease, appropriate measures like mass awareness, health education, better medical facilities, identification \& mass treatment of $T$. solium carriers and restriction on sale of contaminated pork may help to reduce the disease burden in the endemic areas.

\section{Reference}

1. Siddeswari R, Manohar S, Sudarsi B.Cutaneous cysticercosis. J Acad Med Sci 2012;2:132-4.

2. S Gole, G Gole, V Satyanarayana, A Deshpande, S Tati. Cysticercosis At Rare Sites: Our Experience At A Rural Medical College In Andhra Pradesh, India. The Internet Journal of Parasitic Diseases. 2012; 5(1).

3. Agrawal R. Soft tissue cysticercosis study of 21 cases. J Clin Diagn Res. 2012; 6:1669-71.

4. Del Brutto OH. Neurocysticercosis: a review. Sci World J. 2012;2012:159821

5. Nigam JS, Sharma A. Fine needle aspiration cytology of cysticercosis. J Clin Diagn Res. 2013;7:3123-3123.

*Corresponding author:

Dr. Tanima Dwivedi, Senior Resident, Department of Pathology, Institute of Human Behaviour \& Allied Sciences, Delhi-95, India

Phone: +91 0124-4259965

Email: tanimadwivedi@gmail.com

Date of Submission : 21.04.2017

Date of Acceptance : 21.05.2017

Financial or other Competing Interests: None.

Date of Publication : 28.05.2017 